CERN-TH.7210/94

hep-th/9403183

\title{
On the Landau-Ginzburg Realization of Topological Gravities
}

\author{
W. Lerche \\ and \\ A. Sevrin \\ CERN, Geneva, Switzerland
}

\begin{abstract}
We study the equivariant cohomology of a class of multi-field topological Landau-Ginzburg models, and find that such systems carry intrinsic information about $W$-gravity. As a result, we can construct the gravitational chiral ring in terms of LG polynomials. We find, in particular, that the spectrum of such theories seems to be richer than so far expected. We also briefly discuss the BRST operator for non-linear topological $W$-gravity.
\end{abstract}

CERN-TH.7210/94

March 1994 


\section{Introduction}

By now it is well-known [1] 3] that certain systems of conformal matter coupled to $2 d$-gravity have an equivalent description in terms of topological Landau-Ginzburg theory [4]. More precisely, the minimal models of type $(1, t)$ coupled to gravity are closely related to the twisted [5,6] $N=2$ superconformal minimal models of type $A_{k+1}$, where $t=k+2$. In particular, the dynamics of both systems are governed [7, 叉] by the same KdV-type of integrable systems. This, in effect, allows to use $N=2$ Landau-Ginzburg methods to obtain further insight in the matter-gravity systems, and to explicitly compute various correlation functions.

There is, however, an important difference between the matter-gravity system and the topological minimal model: the spectrum of of $A_{k+1}$ is given by the chiral primary ring

$$
\mathcal{R}_{x}=\left\{1, x, x^{2}, \ldots, x^{k}\right\},
$$

whereas for the chiral ground ring of the matter-gravity system one has infinitely many more physical operators:

$$
\mathcal{R}_{x, \gamma}=\mathcal{R}_{x} \otimes\left\{\left(\gamma^{0}\right)^{l}, l=0,1,2, \ldots\right\} .
$$

This is also the spectrum of an a priori different system, namely of topological minimal matter $A_{k+1}$ coupled [9] to topological gravity [10]. From this viewpoint, one may interpret $\left(\gamma^{0}\right)^{l}$ as gravitational descendants and call $\mathcal{R}_{x, \gamma}$ an gravitationally extended chiral ring.

One can actually modify the cohomological definition of the topological matter theory such that its spectrum becomes precisely $\mathcal{R}_{x, \gamma}$ [2,11]. That is, upon requiring equivariant cohomology, which amounts to imposing the condition that the anti-ghost $b$ annihilates the physical states [10,12], the gravitational descendants $\left(\gamma^{0}\right)^{l}$ become physical in the modified theory. This means, however, that the structure of the gravitational sector must, in some way, already be built in the topological matter model.

Indeed, in the Landau-Ginzburg formulation of topological minimal matter [13], where $x$ in (1.1) is viewed as the LG field (with superpotential $W(x)=x^{k+2}$ ), the gravitational descendants can be very simply constructed in terms of $x$ as well [14]:

$$
\left(\gamma^{0}\right)^{l} x^{i}=\sigma_{l}\left(x^{i}\right)=x^{i+(k+2) l}, \quad x^{(l+1)(k+2)-1} \equiv 0
$$

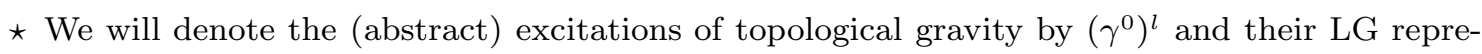
sentatives by $\sigma_{l}$. 
(we also exhibited here the null states of the theory). In more technical terms, the BRST cohomology of the topological matter-gravity system has representatives that lie entirely in the matter sector [11].

It is the gravitational ring structure (1.3), depicted in Fig.1, that is common to many algebraic features of the theory, like for example the spectrum of KdV flows. One of our concerns is to analyze the corresponding structure of more complicated versions of $2 d$ gravity in connection with Landau-Ginzburg models. In essence, gravity models with additional symmetries lead to LG models with more basic fields and more types of gravitational excitations. In particular, we want to understand the gravitational chiral rings that are implicitly built in more complicated LG models. The rule, valid at least for a large class of theories, seems to be that one gets a new type of gravitational descendant generator for each new LG field, as well as an independent supercurrent generator of the extended topological symmetry algebra [2,15]. What we hope for to find, ultimately, is an intrinsic and generic relationship between $N=2$ Landau-Ginzburg theory, variations of topological gravity and integrable systems [15].

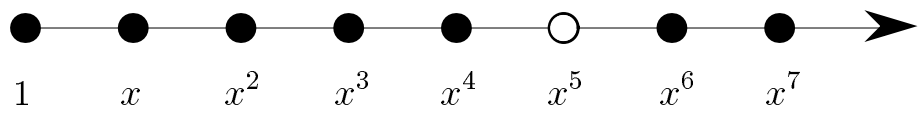

Fig. 1 Chiral ring spectrum and pattern of KdV flows in the topological minimal model of type $A_{5}$ coupled to gravity. The open dot describes a null field.

In the present paper, we consider as next step in this project the question, how the extended gravitational sector can be recovered from within a more general, multivariable LG theory.

Specifically, we will focus on the $W_{3}$ extension of gravity coupled [16] to matter of type $(1, t)$. We do this not because $W_{3}$ matter-gravity systems would be particularly important, but because these systems are the simplest possible extensions of ordinary gravity theories, the study of which ought to provide insight into the general case. It is known [2] that such systems have an analogous close connection to topological $W_{3}$ minimal models, which are just the (twisted) $N=2$ coset models [17 based on 
$\frac{S U(3)_{k}}{U(2)}($ where $t=k+3$ ), and which have a well-understood [18, 19] Landau-Ginzburg description as well.

In the next section, we will study the $W_{3}$ symmetry properties of such LG models. We then couple them to non-linear topological $W_{3}$-gravity, which we define in terms of quantum Hamiltonian reduction of $O S p(6 \mid 4)$ and which is different from the kinds of linear $W_{3}$-gravity discussed so far in the literature [20]. Though we did not fully succeed in explicitly writing down the complete BRST operator, we will go sufficiently far to be able to investigate the relevant part of the equivariant cohomology of the matter-gravity system. We will see that the non-trivial cohomology can indeed be represented in terms of the Landau-Ginzburg sector alone, and we will be able to write the observables of topological $W$-gravity in terms of LG polynomials. As it will be discussed in the conclusions, we find that there are actually more physical operators than it was expected from previous work. Finally, in the appendix we will explain our superspace conventions.

\section{$2 \mathrm{~W}_{3}$ symmetry in $\mathrm{N}=2$ Landau-Ginzburg models}

Following in spirit 21,22, we will describe the topological $W_{3}$ matter sector in terms "almost" free LG fields. * We find that the formulae become particularly compact if we choose chiral superfields $X_{i}, \Psi_{i} i=1,2$, as follows: ${ }^{\dagger}$

$$
\begin{aligned}
& X_{i}=x_{i}+\theta^{-} \psi_{i}^{+}-\frac{1}{2} \theta^{+} \theta^{-} \partial x_{i} \\
& \Psi_{i}=\bar{\psi}_{i}^{+}+\theta^{-} \partial x_{i}^{*}-\frac{1}{2} \theta^{+} \theta^{-} \partial \bar{\psi}_{i}^{+} .
\end{aligned}
$$

The component fields are the usual building blocks of topological LG models [4], and are nothing but a collection of ghost systems in disguise: $x=\beta, \psi^{+}=b, \bar{\psi}^{+}=$ $c, \partial x^{*}=\gamma$. In computing operator products, one can safely ignore the superpotential, since it gives rise only to soft contributions. Therefore, we are effectively dealing with a free theory,

$$
X_{i}\left(Z_{1}\right) \Psi_{j}\left(Z_{2}\right)=\delta_{i j} \frac{\theta_{12}^{-}}{z_{12}}
$$

* After we had finished the computations for this section, we received the paper 23 which has some overlap with our results.

$\dagger$ We will be considering in this paper only the left-moving part of the theory. For the rightmovers, analogous formulae hold. 
with super stress tensor [21,22]

$$
\mathcal{T}_{m}(X, \Psi)=\sum_{i=1}^{2}\left[\omega_{i} X_{i}\left(D_{-} \Psi_{i}\right)+\left(\omega_{i}-1\right)\left(D_{-} X_{i}\right) \Psi_{i}\right]
$$

(relevant for us is, of course, the topologically twisted version [5],6], which is considered later). Above, $\omega_{i}=i / t$ are the $U(1)$ charges of the fields $X_{i}$. The stress tensor gives rise to a central charge of

$$
c_{m}=6\left(\frac{t-3}{t}\right),
$$

and this is precisely the central charge of the $N=2$ coset models $\mathrm{CP}_{2, k}$ [17] based on $\frac{S U(3)_{k}}{U(2)}$, if one sets

$$
t=k+3
$$

These models are well-known to exhibit an $N=2 W_{3}$ superconformal symmetry [24], and indeed we can construct with the above building blocks the following primary, spin-2 supercurrent:

$$
\begin{aligned}
\mathcal{W}_{m} & \left(X_{i}, \Psi_{i}\right)= \\
& \alpha_{m}\left\{\frac{\left(2-9 t+2 t^{2}\right)(t-1)}{(t-4) t(2+t)}\left(D_{-} X_{1}\right)\left(D_{-} X_{2}\right) \Psi_{1} \Psi_{2}-\frac{(5 t-18)}{(t-4)(2+t)}\left(D_{-} X_{1}\right) X_{2}\left(D_{-} \Psi_{1}\right) \Psi_{2}\right. \\
& -\frac{(t-1)}{(t-4) t}\left(D_{-} X_{1}\right) X_{2} \Psi_{1}\left(D_{-} \Psi_{2}\right)-\frac{(t-5)(2 t-1)}{2 t(2+t)}\left(D_{-} X_{1}\right) \partial \Psi_{1}-\frac{(t-1)}{t}\left(D_{-} X_{2}\right) \partial \Psi_{2} \\
& +\frac{(t-1)\left(2 t^{2}-5 t-4\right)}{2(t-4) t(2+t)}\left(D_{-} \partial X_{1}\right) \Psi_{1}+\frac{\left(2-4 t+t^{2}\right)(t-2)}{(t-4) t(2+t)}\left(D_{-} \partial X_{2}\right) \Psi_{2}+\frac{(t-5)}{2 t(2+t)} X_{1}\left(D_{-} \partial \Psi_{1}\right) \\
& -\frac{(t-1)(3 t-8)}{(t-4) t(2+t)} X_{1}\left(D_{-} X_{1}\right) \Psi_{1}\left(D_{-} \Psi_{1}\right)+\frac{2-9 t+2 t^{2}}{(t-4) t(2+t)} X_{1}\left(D_{-} X_{2}\right)\left(D_{-} \Psi_{1}\right) \Psi_{2} \\
& +\frac{(3 t-8)}{2(t-4) t(2+t)}\left(X_{1}\right)^{2}\left(D_{-} \Psi_{1}\right)^{2}+\frac{1}{(t-4) t} X_{1} X_{2}\left(D_{-} \Psi_{1}\right)\left(D_{-} \Psi_{2}\right)+\frac{1}{t} X_{2}\left(D_{-} \partial \Psi_{2}\right) \\
& -\frac{(t-2)}{(t-4) t} X_{2}\left(D_{-} X_{2}\right) \Psi_{2}\left(D_{-} \Psi_{2}\right)+\frac{1}{(t-4) t}\left(X_{2}\right)^{2}\left(D_{-} \Psi_{2}\right)^{2} \\
& \left.-\frac{(1+t)\left(2 t^{2}-5 t-4\right)}{2 t(t-4)(2+t)} \partial X_{1}\left(D_{-} \Psi_{1}\right)-\frac{\left(2-4 t+t^{2}\right)}{(t-4) t} \partial X_{2}\left(D_{-} \Psi_{2}\right)\right\}+A \widetilde{\mathcal{W}}_{m}
\end{aligned}
$$

where

$$
\widetilde{\mathcal{W}}_{m}=X_{2}\left(D_{-} \Psi_{1}\right)^{2}+(1-t)\left(D_{-} X_{2}\right) \Psi_{1}\left(D_{-} \Psi_{1}\right)
$$

and where $A$ is a free parameter. Together with $\mathcal{T}_{m}, \mathcal{W}_{m}$ generates the $N=2 W_{3}$ superconformal algebra (A.4). Taking $\alpha_{m} \equiv \sqrt{\frac{(t-4)(t+2)}{(2 t-3)(5 t-18)}}$ gives the normalization: $\mathcal{W}_{m} \cdot \mathcal{W}_{m} \sim \frac{1}{6} c_{m} \frac{1}{(z-w)^{4}}+\ldots$

The full algebra that $\mathcal{T}_{m}$ and $\mathcal{W}_{m}$ generate is actually somewhat larger, due to the presence of the free parameter $A$ in (2.6). Indeed $\mathcal{T}_{m}, \widetilde{\mathcal{W}}_{m}$ form a separate linear 
$N=2 W_{3}$ algebra with $\widetilde{\mathcal{W}}_{m} \cdot \widetilde{\mathcal{W}}_{m} \sim 0$, so that the full algebra is a semi-direct product of a linear with a non-linear $N=2 W_{3}$ algebra. However, the chiral algebra currents must commute with the screening operators of the theory, a quantum version of the requirement that the currents be conserved, and this is what fixes $A$. In the present $N=2$ Landau-Ginzburg theories describing the models $\mathrm{CP}_{2, k}$, the relevant screeners [21,22] are given in terms of the superpotentials: $Q_{W}=\frac{1}{2 \pi i} \oint d z d \theta^{-} W_{k}\left(X_{1}, X_{2}\right)$. Requiring

$$
\frac{1}{2 \pi i} \oint d z d \theta^{-} W_{k}\left(X_{1}, X_{2}\right)(Z) \cdot \mathcal{T}_{m}=0
$$

just yields the usual condition for $W_{k}$ to be quasi-homogenous: $W_{k}=\sum \omega_{i} x_{i} \partial_{i} W_{k}$. But requiring in addition

$$
\frac{1}{2 \pi i} \oint d z d \theta^{-} W_{k}\left(X_{1}, X_{2}\right)(Z) \cdot \mathcal{W}_{m}=0
$$

gives

$$
A=\frac{\alpha_{m}}{t} \frac{(18-5 t)}{(t-4)(2+t)},
$$

and produces a large list of additional differential equations for the superpotentials $W_{k}$. A typical equation is, for example

$$
\begin{aligned}
& \left\{(t-4)(2+t) \frac{\partial}{\partial x_{2}}+(3 t-8) x_{2} \frac{\partial^{2}}{\partial x_{2}^{2}}+\right. \\
& \left.\quad(4 t-13) x_{1} \frac{\partial^{2}}{\partial x_{1} \partial x_{2}}+(5 t-18) \frac{\partial^{2}}{\partial x_{1}{ }^{2}}\right\} W_{k}\left(x_{1}, x_{2}\right)=0 .
\end{aligned}
$$

The LG superpotentials of the coset models $\mathrm{CP}_{2, k}$ are well-known [18,25,26], and can be compactly characterized by the following generating function [26]:

$$
-\log \left[\sum_{i=1}^{2}(-\lambda)^{i} x_{i}\right]=\sum_{k=-2}^{\infty} \lambda^{k+3} W_{k}\left(x_{1}, x_{2}\right)
$$

Using identities like [15]

$$
\begin{aligned}
W_{k}\left(x_{1}, x_{2}\right)= & \frac{1}{t}\left(2 \frac{\partial}{\partial x_{1}}+x_{1} \frac{\partial}{\partial x_{2}}\right) W_{k+1}\left(x_{1}, x_{2}\right) \\
\frac{\partial}{\partial x_{1}} W_{k}\left(x_{1}, x_{2}\right)= & \left(x_{1} \frac{\partial}{\partial x_{1}}+x_{2} \frac{\partial}{\partial x_{2}}\right) W_{k-1}\left(x_{1}, x_{2}\right) \\
\frac{\partial}{\partial x_{2}} W_{k}\left(x_{1}, x_{2}\right)= & -\frac{\partial}{\partial x_{1}} W_{k-1}\left(x_{1}, x_{2}\right), \\
& -5-
\end{aligned}
$$


we find that all equations are solved precisely by the superpotentials $W_{k}$. This proves that the models $\mathrm{CP}_{2, k}$ are indeed associated with the "correct" points in the LG moduli spaces that are determined in terms of the cohomology of grassmannians [18, 25, 19, 26].

The explicit form of the $N=2 W_{3}$ symmetry currents allows us to choose a convenient, distinguished basis for the LG polynomials, namely the basis of eigenstates of $V_{0} \equiv \int z \mathcal{W}_{m}$. We find that it is simply given by

$$
\Phi^{l, m}\left(x_{1}, x_{2}\right)=x_{2}{ }^{m}\left(\frac{\partial}{\partial x_{1}} W_{l-2}\left(x_{1}, x_{2}\right)\right) .
$$

These specific polynomials [15] can also be associated with the weights of the Brepresentation of $S U(k+2)$. The chiral primary ring of the matter model $\mathrm{CP}_{2, k}$ [18, 19] in this basis is then

$$
\mathcal{R}_{x}^{(k)}=\left\{\Phi^{l, m}\left(x_{1}, x_{2}\right), \quad m+l \leq k\right\} .
$$

We will show later how the $\Phi^{l, m}$ with $m+l>k$ can be used to describe the $W$-gravity descendants as well.

The $U(1)$ quantum numbers of the ring elements are

$$
J_{0}\left(\Phi^{l, m}\right)=\frac{1}{t}(l+2 m),
$$

and it is straightforward to compute the $V_{0}$ quantum numbers for any given $\Phi^{l, m}$. In particular, we have

$$
\begin{aligned}
& V_{0}\left(\Phi^{1,0} \equiv x_{1}\right)=\alpha_{m} \frac{5-t}{2 t(t+2)} \\
& V_{0}\left(\Phi^{0,1} \equiv x_{2}\right)=-\alpha_{m} \frac{1}{t} .
\end{aligned}
$$

This indeed coincides with the $V_{0}$ quantum numbers one gets from the explicit expressions [2] of the ground ring generators $x_{1}, x_{2}$ of $(1, t)$-type $W_{3}$-matter-gravity systems, by using the current $V$ given in eq. (2.31) of ref. [2]. This is another confirmation that the topologically twisted models $\mathrm{CP}_{2, k}$ are, essentially, equivalent to these systems.

We now turn to the topologically twisted version of the matter theory. Starting from the $N=2$ structure, one obtains the chiral algebra of the topological matter system by twisting the $N=2$ currents. In superspace, these currents are simply given 
by $\left.D_{-} \mathcal{T}_{m}\right|_{\theta^{-}=0}$ and $\left.D_{-} \mathcal{W}_{m}\right|_{\theta^{-}=0}$. Each of them describes a BRST doublet with the BRST charge given by

$$
Q_{S}=\frac{1}{2 \pi i} \oint d z d \theta^{-} \mathcal{T}_{m}
$$

From Eq. (A.4), one deduces that that the algebra generated by $\left.D_{-} \mathcal{T}_{m}\right|_{\theta^{-}=0}$ and $\left.D_{-} \mathcal{W}_{m}\right|_{\theta^{-}=0}$ indeed closes, but in a non-linear way. In fact, a detailed inspection reveals that the structure functions do depend on the full $N=2$ currents and not only on the topological currents. In the next section we will describe the coupling the matter system to topological gravity.

\section{Non-linear topological $\mathrm{W}_{3}$-gravity}

In [10], topological gravity was constructed from gauge fixing a theory with trivial action of the $d=2$ Poincare group, a contraction of $S l(2)$. The resulting field content was given by Liouville fields $\pi$ and $\phi$, fermions $\psi$ and $\chi$ and ghosts $b, c, \beta$ and $\gamma$. Several generalizations to topological $W_{3}$-gravity were made in [20] by starting from a gauge theory of some contraction of $S l(3)$. These approaches were however unsatisfying in the sense that the resulting twisted $N=2 W_{3}$ algebra had a linear nature: the OPE of $D_{-} \mathcal{W}$ with itself vanished. On the other hand, from our experience with non-critical $W$-strings [16] we rather expect the Liouville sector to have the same type of chiral algebra as the matter sector. Though it might very well be that the full non-linear $N=2 W_{3}$ algebra arises from the approach of [20] by making a different gauge choice, we do not pursue this approach here.

Instead, motivated by a hidden, doubly twisted $N=4$ superconformal symmetry in topological gravity [27], we will rather define non-linear topological $W$-gravity via quantum Hamiltonian reduction of a WZW model; by general results of Hamiltonian reduction [28], such a theory is consistent by construction, and the existence of a quantum BRST operator is automatic. As will be explained below, this approach indeed implies the existence of a non-linear $N=2 W$-algebra in the Liouville sector. * Therefore, we start by directly constructing the $N=2 W_{3}$ algebra out of the field content of refs. [20]. This essentially amounts to doubling the fields appearing in

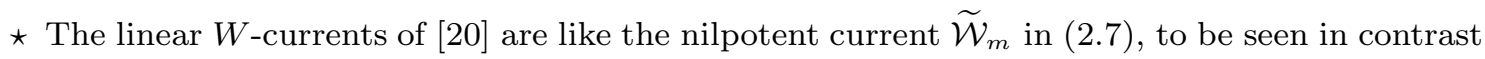
with the non-linear current $\mathcal{W}_{m}$ in (2.6). 
ordinary topological gravity. Again $N=2$ superspace provides the most economical way to do so, and we thus introduce free chiral superfields $\Pi_{i}$ and $\Psi_{i}, i=1,2$,

$$
\begin{aligned}
& \Pi_{i}=\pi_{i}+\theta^{-} \partial \chi_{i}-\frac{1}{2} \theta^{+} \theta^{-} \partial \pi_{i} \\
& \Phi_{i}=\psi_{i}+\theta^{-} \partial \phi_{i}-\frac{1}{2} \theta^{+} \theta^{-} \partial \psi_{i}
\end{aligned}
$$

with OPE's

$$
\Pi_{i}\left(Z_{1}\right) \Phi_{j}\left(Z_{2}\right)=\frac{1}{z_{12}} \delta_{i j} \theta_{12}^{-} .
$$

The super stress tensor of the ordinary topological Liouville sector (before twisting) is

$$
\mathcal{T}_{l, 1}=-\Phi_{1}\left(D_{-} \Pi_{1}\right)+q\left(D_{-} \Phi_{1}\right)+q \partial \Pi_{1}
$$

which has central charge $c_{l, 1}=3\left(1+2 q^{2}\right)$. It turns out that the complete $N=2 W_{3}$ algebra can be realized in terms of $\mathcal{T}_{l, 1}$ alone, together with the extra $W_{3}$ Liouville fields $\Pi_{2}, \Phi_{2}$ (completely analogous to the free field realization of the ordinary $W_{3^{-}}$ algebra [29]). In particular, the total Liouville super stress tensor is

$$
\mathcal{T}_{l}=\mathcal{T}_{l, 1}-\Phi_{2}\left(D_{-} \Pi_{2}\right)+q\left(D_{-} \Phi_{2}\right)+2 q \partial \Pi_{2}
$$

whose central charge depends on the background charge parameter $q$ as follows:

$$
c_{l}=6\left(1+3 q^{2}\right)
$$

In addition, we have the following primary, spin-2 supercurrent in the Liouville sector:

$$
\begin{aligned}
& \mathcal{W}_{l}(\Pi, \Phi)= \\
& \quad \alpha_{l}\left\{\frac{1}{2} q^{2} \mathcal{T}_{l, 1}^{\prime}+\frac{2\left(1-2 q^{2}\right)\left(1+3 q^{2}\right)}{10+36 q^{2}}\left(D_{+-} \mathcal{T}_{l, 1}\right)-\frac{\left(8 q^{2}-3\right)}{10+36 q^{2}}\left(\mathcal{T}_{l, 1}\right)^{2}\right. \\
& \quad+\frac{2 q\left(1+5 q^{2}\right)}{5+18 q^{2}} \mathcal{T}_{l, 1}\left(D_{-} \Phi_{2}\right)-\frac{2\left(1+5 q^{2}\right)}{5+18 q^{2}} \mathcal{T}_{l, 1}\left(D_{-} \Pi_{2}\right) \Phi_{2}+\frac{q\left(2 q^{2}-1\right)}{5+18 q^{2}} \mathcal{T}_{l, 1} \partial \Pi_{2} \\
& \quad+\frac{q\left(3+8 q^{2}\right)}{5+18 q^{2}}\left(D_{-} \Pi_{2}\right) \Phi_{2}\left(D_{-} \Phi_{2}\right)+\frac{1+6 q^{2}+12 q^{4}}{5+18 q^{2}}\left(D_{-} \Pi_{2}\right) \partial \Phi_{2}-\frac{q^{2}\left(3+8 q^{2}\right)}{2\left(5+18 q^{2}\right)}\left(D_{-} \Phi_{2}\right)\left(D_{-} \Phi_{2}\right) \\
& \quad+\frac{\left(1+3 q^{2}\right)\left(2 q^{2}-1\right)}{5+18 q^{2}}\left(D_{-} \partial \Pi_{2}\right) \Phi_{2}-q\left(D_{+} \mathcal{T}_{l, 1}\right)\left(D_{-} \Pi_{2}\right)+\frac{\left(1-2 q^{2}\right)\left(1+2 q^{2}\right)}{5+18 q^{2}} \partial \Pi_{2}\left(D_{-} \Phi_{2}\right) \\
& \quad+\frac{q\left(1-2 q^{2}\right)}{5+18 q^{2}} \partial \Pi_{2}\left(D_{-} \Pi_{2}\right) \Phi_{2}+\frac{q^{2}\left(2 q^{2}-1\right)}{5+18 q^{2}} \partial \Pi_{2} \partial \Pi_{2}+\frac{q\left(1-2 q^{2}\right)\left(1+3 q^{2}\right)}{5+18 q^{2}} \partial^{2} \Pi_{2} \\
& \left.\quad-\frac{q\left(1+6 q^{2}+12 q^{4}\right)}{2\left(5+18 q^{2}\right)}\left(D_{-} \partial \Phi_{2}\right)\right\} .
\end{aligned}
$$


which generates, together with $\mathcal{T}_{l}$, the non-linear $N=2 W_{3}$ algebra (A.4). We stress once more that this is unlike the spin-2 Liouville supercurrents discussed in the literature [20], which generate linear $N=2 W_{3}$ algebras and square to zero. Taking $\alpha_{l}=\sqrt{\frac{\left(5+18 q^{2}\right)}{\left(1-2 q^{2}\right)\left(2+3 q^{2}\right)\left(1+4 q^{2}\right)}}$ gives the normalization: $\mathcal{W}_{l} \cdot \mathcal{W}_{l} \sim \frac{1}{6} c_{l} \frac{1}{(z-w)^{4}}+\ldots$ The topological currents are given by $\left.D_{-} \mathcal{T}_{l}\right|_{\theta^{-}=0}$ and $\left.D_{-} \mathcal{W}_{l}\right|_{\theta^{-}=0}$ and generate precisely the same algebra as $\left.D_{-} \mathcal{T}_{m}\right|_{\theta^{-}=0}$ and $\left.D_{-} \mathcal{W}_{m}\right|_{\theta^{-}=0}$.

The ghost system is described by free anti-chiral superfields $(i=1,2)$

$$
\begin{aligned}
& B_{i}=\beta_{i}+\theta^{+} b_{i}-\frac{1}{2} \theta^{+} \theta^{-} \partial \beta_{i} \\
& C_{i}=c_{i}+\theta^{+} \gamma_{i}-\frac{1}{2} \theta^{+} \theta^{-} \partial c_{i} .
\end{aligned}
$$

with

$$
B_{i}\left(Z_{1}\right) C_{j}\left(Z_{2}\right)=\frac{1}{z_{12}} \delta_{i j} \theta_{12}^{+} .
$$

Above, $\left(b_{i}, c_{i}\right)$ are the usual fermionic ghosts with spins $(i+1,-i)$, and $\left(\beta_{i}, \gamma_{i}\right)$ are their superpartners. The ghost number current looks

$$
\mathcal{T}_{g \#}=\sum_{i=1}^{2}\left\{D_{+}\left(C_{i} B_{i}\right)+\left(D_{+} C_{i}\right) B_{i}\right\}
$$

whereas the ghost super stress tensor is given by

$$
\mathcal{T}_{g h}=-\left(D_{+} B_{1}\right) C_{1}-2 B_{1}\left(D_{+} C_{1}\right)-2\left(D_{+} B_{2}\right) C_{2}-3 B_{2}\left(D_{+} C_{2}\right),
$$

with central charge $c_{g h}=-3(3+5)=-24$. In (3.5), the value $q=1$ gives $c_{l}=24$, which cancels the central charge of the topological ghost system, and is the $W_{3}$ analog of the "critical" central charge $c_{l}=9$ of ordinary topological gravity. However, there is no need to cancel the central charge of the ghosts, because in topological gravities the BRST operator squares to zero independently of that; we will thus assume $q=1$ henceforth, even when we will couple in topological matter.

In pure topological $W_{3}$-gravity, the BRST operator has the form

$$
Q_{B R S T}=Q_{S}+Q_{V}
$$

where

$$
Q_{S}=\frac{1}{2 \pi i} \oint d z d \theta^{+}\left[\mathcal{T}_{l}+\mathcal{T}_{g h}\right]
$$


is the total supersymmetry charge, and

$$
Q_{V}=\frac{1}{2 \pi i} \oint d z d \theta^{+}\left[C_{1} D_{-}\left(\mathcal{T}_{l}+\frac{1}{2} \mathcal{T}_{g h}\right)+C_{2}\left(D_{-} \mathcal{W}_{l}+\frac{1}{2} \widehat{\mathcal{W}}_{g h}\right)\right]
$$

The only unknown in (3.13) is the twisted spin-2 ghost current $\widehat{\mathcal{W}}_{g h}=D_{-} \mathcal{W}_{g h}$. It is given to leading order by

$$
\begin{aligned}
\widehat{\mathcal{W}}_{g h} & =3 B_{2} \partial C_{1}+\partial B_{2} C_{1} \\
& -\frac{5 i \sqrt{2}}{\sqrt{69}} B_{1} \partial^{2} C_{1}+\frac{100(31+4 \sqrt{6})}{5175} B_{1} \partial^{3} C_{2}+4 i \frac{\sqrt{2}-3 \sqrt{3}}{\sqrt{69}} \partial B_{2} \partial C_{2}+\frac{10(5 \sqrt{6}-67)}{1725} \partial^{2} B_{1} \partial C_{2} \\
& +\frac{4 i}{\sqrt{23}} \mathcal{T}_{l} B_{2} \partial C_{2}-\frac{10(5 \sqrt{6}-6)}{575} \mathcal{T}_{l} \partial B_{1} \partial C_{2}+\frac{44 i}{5 \sqrt{23}} \mathcal{W}_{l} B_{1} \partial C_{2}+\frac{2(25 \sqrt{6}-94)}{1725} \mathcal{T}_{l}^{\prime} B_{1} \partial C_{2} \\
& +\frac{2 i \sqrt{2}}{\sqrt{69}}\left(D_{-} \mathcal{T}_{l}\right) B_{2}\left(D_{+} C_{2}\right)+\frac{2 i(5 \sqrt{6}-36)}{15 \sqrt{23}}\left(D_{-} \mathcal{W}_{l}\right) B_{1}\left(D_{+} C_{2}\right)+\frac{1004}{1725}\left(D_{+-} \mathcal{T}_{l}\right) B_{1} \partial C_{2} \\
& -\frac{22}{575} \mathcal{T}_{l}^{2} B_{1} \partial C_{2}+\ldots
\end{aligned}
$$

Unfortunately, for technical reasons we have not been able, despite much effort, to compute all terms of $\widehat{\mathcal{W}}_{g h}$. However, we were careful to ensure that our results, and the conclusions we draw from them, do not depend on the extra terms in (3.14). We also found that already with the above, incomplete form of (3.14), many terms cancel in $\left(Q_{B R S T}\right)^{2}$ in a highly non-trivial way (actually, all terms that are independent of the anti-ghosts $B$ and in particular, all non-linear terms that arise from $\left.\left(C_{2} D_{-} \mathcal{W}_{l}\right)^{2}\right)$.

The only feature that is important in the present context is that the full BRST operator really exists at all (and satisfies the identity (4.1) below).

In order to argue so, note that in ordinary topological gravity the presence of two BRST charges $Q_{V}, Q_{S}$ (and thus of two "BRST-ancestors" of the energy-momentum tensor, namely the anti-ghost $b_{1}$ and the fermionic current $G_{-}$), indicates a hidden, doubly twisted $N=4$ superconformal structure. An explicit realization of the theory can be obtained by the Hamiltonian reduction of $O S p(4 \mid 2)$ determined by its maximal regular subalgebra $S l(2 \mid 1)$. The gradation used in the reduction is tuned precisely such that the resulting $N=4$ algebra is doubly twisted. Topological gravity from this Hamiltonian reduction point of view will be discussed in detail in ref.[27].

The obvious question in the present context is whether the $N=4$ superconformal structure of topological gravity extends to topological $W$-gravity. It is clear that a necessary requirement for this is the existence of an $N=4 W_{n}$ algebra that contains the $N=2 W_{n}$-algebra. In order to track down such an algebra, Hamiltonian reduction is again the method of choice. The $N=2 W_{n}$ algebra is obtained from a reduction 
of $S l(n \mid n-1)$ determined by the principal embedding of $S l(2 \mid 1)$ [24]. Analyzing possible embeddings of $S l(n \mid n-1)$ in supergroups [30], one arrives at the conclusion that only one natural candidate for an $N=4 W_{n}$ algebra exists: $\operatorname{OSp}(2 n \mid 2(n-1))$, which has $S l(n \mid n-1)$ as a maximal regular subalgebra; see Fig.2. The embedding $S l(2 \mid 1) \hookrightarrow O S p(2 n \mid 2(n-1))$ is the principal embedding in the maximal regular $S l(n \mid n-$ 1) subalgebra. Because of this particular choice of the embedding of $S l(2 \mid 1)$, we have already the guarantee that the superconformal algebra contains the $N=2 W_{n}$ algebra as a subalgebra.

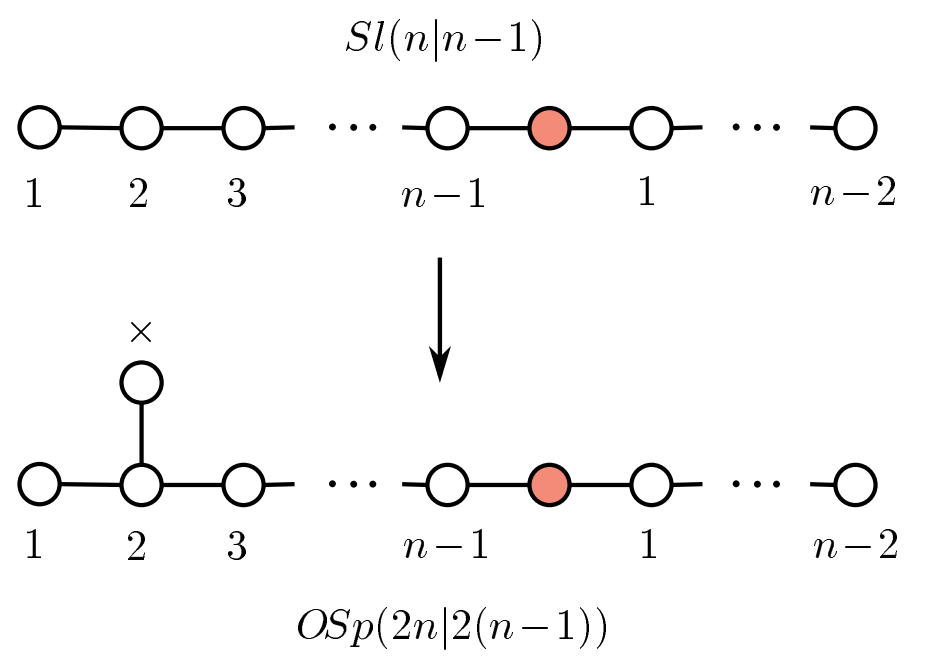

Fig. 2 The Dynkin diagrams of $S l(n \mid n-1)$ and $O S p(2 n \mid 2(n-1))$. The embedding of $S l(n \mid n-1)$ is obtained by removing the dot denoted by $\times$ in the diagram of $\operatorname{OSp}(2 n \mid 2(n-1))$.

Using the general framework set up in [28], one finds that the resulting current algebra consists of unconstrained $N=2$ superfields ${ }^{\star}$ of dimension $1,2, \ldots, n-1$, which generate an $N=2 W_{n}$ algebra. Besides these, one has a dimension 0 superfield (which in analogy with the ordinary $N=4$ algebra only appears through its $D_{+}$ or $D_{-}$derivative) and couples of fields of dimension $(2 k+1) / 2$ where either $k=$ $0,2, \ldots,(n-2)$ for $n$ even or $k=1,3, \ldots,(n-2)$ for $n$ odd. The algebra has indeed four supersymmetry currents of dimension $3 / 2$. However, in order to call this an $N=4$ algebra, we also need that the supercharges can be viewed as "square

* In order to complete the "short" $N=2$ multiplets, some free fermions and a free scalar field have to be added. This is the reverse of the mechanism discussed in [31]. 
roots" of the translations. A sufficient condition for this is that the $S l(2)$ subalgebra of $O S p(2 n \mid 2(n-1))$, which yields the energy-momentum tensor in the reduction, together with the two angular momentum $1 / 2$ multiplets (which give rise to the two other dimension 3/2 supercurrents), generate an $S l(2 \mid 1)$ subalgebra. We verified that this is indeed the case for $n=2$ and 3 and expect it to be valid for arbitrary $n$.

Thus, all ingredients for performing a double twist and thus obtaining topological $W_{n}$-gravity are indeed present. Though we leave the detailed investigation of these matters for future work [27], the arguments just given almost prove the existence of $Q_{V}$ in topological $W$-gravity (at the quantum level). In view of the great computational difficulties we encountered in directly constructing $Q_{V}$, it might very well be that the above discussed method provides the only economic way to obtain $Q_{V}$ explicitly.

In order to couple in additional matter, one may follow the philosophy of [16]. There, at least for $W_{3}$ gravity, it was argued that coupling matter to a non-linear algebra is possible provided the combined matter-gravity sector has a closed (possibly soft) gauge algebra at the classical level. The structure of the classical $N=2 W_{3}$ algebra is schematically given by

$$
\begin{aligned}
\mathcal{T} \mathcal{T} & =[\mathcal{T}] \\
\mathcal{T} \mathcal{W} & =[\mathcal{W}] \\
\mathcal{W} \mathcal{W} & =[\mathcal{T} \mathcal{T} \mathcal{T}]+[\mathcal{T} \mathcal{W}] .
\end{aligned}
$$

If we now introduce the total currents $\mathcal{T}_{\text {tot }}=\mathcal{T}_{l}+\mathcal{T}_{m}$ and $\mathcal{W}_{\text {tot }}=\mathcal{W}_{l}-\mathcal{W}_{m}$, we find that the classical algebra indeed closes. The only non-trivial OPE is the one of $\mathcal{W}_{\text {tot }}$ with itself, which has the structure:

$$
\begin{aligned}
\mathcal{W}_{\text {tot }} \mathcal{W}_{\text {tot }}= & {\left[\left(\mathcal{T}_{m} \mathcal{T}_{m}-\mathcal{T}_{m} \mathcal{T}_{l}+\mathcal{T}_{l} \mathcal{T}_{l}\right) \mathcal{T}_{\text {tot }}\right]+} \\
& \frac{1}{2}\left[\left(\mathcal{T}_{l}-\mathcal{T}_{m}\right) \mathcal{W}_{\text {tot }}\right]+\frac{1}{2}\left[\left(\mathcal{W}_{l}+\mathcal{W}_{m}\right) \mathcal{T}_{\text {tot }}\right]
\end{aligned}
$$

We thus expect the BRST charge to have the form:

$$
Q_{V}=\frac{1}{2 \pi i} \oint d z d \theta^{+}\left[C_{1} D_{-}\left(\mathcal{T}_{l}+\mathcal{T}_{m}+\frac{1}{2} \mathcal{T}_{g h}\right)+C_{2}\left(D_{-} \mathcal{W}_{l}-D_{-} \mathcal{W}_{m}+\frac{1}{2} \widehat{\mathcal{W}}_{g h}\right)\right],
$$

where $\widehat{\mathcal{W}}_{g h}$ is very similar to eq. (3.14), except for some multiplicative renormalizations and the fact that the Liouville-dependent parts will be replaced by the structure functions (cf., (3.16)) of the combined matter-gravity gauge algebra. Note, though, that we do not need in the following the exact form of (3.17). The point is that the Liouville sector is necessary only to obtain a covariant formulation [32], and can in principle be decoupled. Indeed, it does not play any role in our subsequent discussion of equivariant cohomology. Therefore, for our purposes, we can effectively take as BRST operator the expression (3.13), with $\mathcal{T}_{l}$ and $\mathcal{W}_{l}$ replaced by $\mathcal{T}_{m}$ and $-\mathcal{W}_{m}$ of eqs. (2.3), (2.6), respectively. 


\section{Equivariant cohomology in the topological LG system}

In analogy to ref. [11], we introduce the following homotopy operator,

$$
S=e^{\frac{1}{2 \pi i} \oint d z\left(c_{1} \widetilde{G}^{-}+c_{2} \widetilde{U}^{-}\right)}
$$

where

$$
\begin{aligned}
& \widetilde{G}^{-}=G_{m}^{-}+\frac{1}{2} G_{g h}^{-} \\
& \widetilde{U}^{-}=-U_{m}^{-}+\frac{1}{2} U_{g h}^{-},
\end{aligned}
$$

with $U^{-}=\left.D_{-} \mathcal{W}\right|_{\theta^{+}, \theta^{-}=0}$. It is straightforward, but tedious to check that $S$ has the crucial property ${ }^{\star}$

$$
S\left(Q_{S}+Q_{V}\right) S^{-1}=Q_{S}
$$

which means that the cohomology of the full BRST operator is isomorphic to the cohomology of the supersymmetry charge, $Q_{S} \equiv \frac{1}{2 \pi i} \oint\left(G_{m}^{+}+G_{g h}^{+}\right)$. This, ultimately, will imply that the cohomology of the topological matter-gravity system has representatives purely in the matter sector.

Specifically, consider some polynomial in the LG fields that is proportional to the vanishing relations,

$$
\Phi\left(x_{1}, x_{2}\right)=\sum_{i=1}^{2} P_{i}^{\Phi}\left(x_{1}, x_{2}\right) \frac{\partial}{\partial x_{i}} W\left(x_{1}, x_{2}\right)
$$

which is a null field in the topological matter model. Using $\delta_{s} \bar{\psi}_{i}^{+}=\frac{1}{2} \partial_{x_{i}} W$ for the BRST variation in the topological matter model [4, 21, 11], one can write

$$
\begin{aligned}
\Phi\left(x_{1}, x_{2}\right) & =\frac{1}{2}\left\{Q_{s}, \sum_{i=1}^{2} \bar{\psi}_{i}^{+} P_{i}^{\Phi}\left(x_{1}, x_{2}\right)\right\} \\
& =: \frac{1}{2}\left\{Q_{s}, \Lambda^{\Phi}\right\} .
\end{aligned}
$$

Whether $\Phi$ is a null operator or not after coupling to topological gravity, depends thus on whether $\Lambda^{\Phi}$ is a physical operator or not. The point is that in equivariant cohomology, the physical states are required to be killed by the zero modes of the

$\star$ We checked this identity up to the order for which the approximation of the BRST operator (3.13), (3.14) is reliable. It seems to trace back to an automorphism of $O S p(6 \mid 4)$, as will be discussed elsewhere [27]. 
anti-ghosts, and this means for $W_{3}$ gravity: $\left(b_{1}\right)_{0}^{-}|\Phi\rangle \equiv\left(b_{1}-\bar{b}_{1}\right)_{0}|\Phi\rangle=0,\left(b_{2}\right)_{0}^{-}|\Phi\rangle \equiv$ $\left(b_{2}-\bar{b}_{2}\right)_{0}|\Phi\rangle=0$. These conditions are important for defining correlation functions of topological gravity unambiguously [20,33]. Like for ordinary topological gravity [11], one can employ the similarity transformation $S$ to rotate the states into the "matter" picture, $|\Phi\rangle_{m}=S|\Phi\rangle$. Under this transformation, one has:

$$
\begin{aligned}
& S\left(b_{1}\right)_{0} S^{-1}=\left(b_{1}+G_{t o t}^{-}\right)_{0} \\
& S\left(b_{2}\right)_{0} S^{-1}=\left(b_{2}+U_{t o t}^{-}\right)_{0},
\end{aligned}
$$

so that in the matter picture the physical state conditions are

$$
\begin{aligned}
Q_{S}|\Phi\rangle_{m} & \cong 0 \\
\left(b_{1}+G_{t o t}^{-}\right)_{0}^{-}|\Phi\rangle_{m} & \cong 0 \\
\left(b_{2}+U_{t o t}^{-}\right)_{0}^{-}|\Phi\rangle_{m} & \cong 0,
\end{aligned}
$$

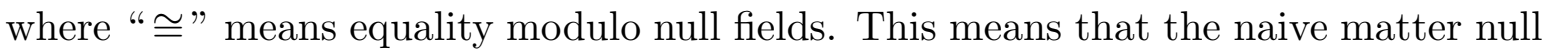
field $\Phi\left(x_{1}, x_{2}\right)$ in (4.5) remains null after coupling to topological gravity precisely if

$$
\begin{aligned}
& \left\{\left(b_{1}+G_{\text {tot }}^{-}\right)_{0}^{-}, \Lambda^{\Phi}\right\} \cong 0 \\
& \left\{\left(b_{2}+U_{\text {tot }}^{-}\right)_{0}^{-}, \Lambda^{\Phi}\right\} \cong 0 .
\end{aligned}
$$

Here, only the matter parts in $G_{t o t}^{-}, U_{\text {tot }}^{-}$can contribute, and the matter contributions from $U_{g h}^{-}$can be seen not to yield any additional information. Therefore, the computation boils down to determining

$$
\begin{aligned}
& \mathcal{K}_{1}(\Phi)=\left\{G_{m, 0}^{-}, \Lambda^{\Phi}\right\} \\
& \mathcal{K}_{2}(\Phi)=\left\{U_{m, 0}^{-}, \Lambda^{\Phi}\right\} .
\end{aligned}
$$

This involves only quantities that pertain to the topological matter theory. Using the component forms

$$
\begin{aligned}
& G_{m}^{-}=\sum \psi_{i}^{+} \partial x_{i}^{*} \\
& U_{m}^{-}=\alpha_{m}\left\{2 \psi_{1}^{+} \psi_{2}^{+} \bar{\psi}_{2}^{+} \partial x_{1}^{*}+\frac{18-5 t}{(t-4)(t+2)} \psi_{2}^{+} \partial x_{1}^{* 2}+\frac{3 t-8}{(t-4)(t+2)} \psi_{1}^{+} x_{1} \partial x_{1}^{* 2}\right. \\
& -\frac{2(t-5)(t-1)}{(t-4)(t+2)} \psi_{1}^{+} \psi_{2}^{+} \bar{\psi}_{1}^{+} \partial x_{2}^{*}-\frac{2(t-5)}{(t-4)(t+2)} \psi_{2}^{+} x_{1} \partial x_{1}^{*} \partial x_{2}^{*}+\frac{2(3 t-8)}{(t-4)(t+2)} \psi_{1}^{+} x_{2} \partial x_{1}^{*} \partial x_{2}^{*} \\
& \left.+\frac{1}{t-4} \psi_{2}^{+} x_{2} \partial x_{2}^{* 2}-\frac{2 t^{2}-5 t-4}{(t-4)(t+2)} \partial x_{1}^{*} \partial \psi_{1}^{+}-\frac{2\left(2-4 t+t^{2}\right)}{(t-4)(t+2)} \partial x_{2}^{*} \partial \psi_{2}^{+}+\frac{t-5}{t+2} \psi_{1}^{+} \partial^{2} x_{1}^{*}+\psi_{2}^{+} \partial^{2} x_{2}^{*}\right\} \text {, }
\end{aligned}
$$

one can then rewrite the conditions for $\Phi\left(x_{1}, x_{2}\right)$ to be null in the following way: 


$$
\begin{aligned}
\mathcal{K}_{1}(\Phi) & =\frac{1}{2 \pi i} \oint d z z G_{m}^{-}(z) \cdot \Lambda^{\Phi}=\sum_{i=1}^{2} \frac{\partial}{\partial x_{i}} P_{i}^{\Phi}\left(x_{1}, x_{2}\right) \cong 0 \\
\mathcal{K}_{2}(\Phi) & =\frac{1}{2 \pi i} \oint d z z^{2} U_{m}^{-}(z) \cdot \Lambda^{\Phi}=\frac{\alpha_{m}}{(t-4)(t+2)} \times \\
& \left\{\left[\left(t^{2}+4 t-24\right) \frac{\partial}{\partial x_{1}}+2(3 t-8) x_{2} \frac{\partial^{2}}{\partial x_{1} \partial x_{2}}+(3 t-8) x_{1} \frac{\partial^{2}}{\partial x_{1}^{2}}\right] P_{1}^{\Phi}\left(x_{1}, x_{2}\right)\right. \\
+ & {\left[\left(t^{2}-6 t+12\right) \frac{\partial}{\partial x_{2}}+(t+2) x_{2} \frac{\partial^{2}}{\partial x_{2}^{2}}-2(t-5) x_{1} \frac{\partial^{2}}{\partial x_{1} \partial x_{2}}\right.} \\
& \left.\left.-(5 t-18) \frac{\partial^{2}}{\partial x_{1}^{2}}\right] P_{2}^{\Phi}\left(x_{1}, x_{2}\right)\right\} \cong 0 .
\end{aligned}
$$

Obviously, the operators $\mathcal{K}_{i}(\Phi)$ map between LG polynomials, and more specifically, one has the following system of descent equations on the space of polynomials:

$$
\begin{array}{cc}
\Phi=: & \Phi_{(0,0)}+\sum P_{i,(0,0)}^{\Phi} \frac{\partial}{\partial x_{i}} W_{k} \\
\vdots & \vdots \\
\mathcal{K}_{1}\left(\Phi_{\left(n_{1}, n_{2}\right)}\right)=: & \Phi_{\left(n_{1}+1, n_{2}\right)}+\sum P_{i,\left(n_{1}+1, n_{2}\right)}^{\Phi} \frac{\partial}{\partial x_{i}} W_{k} \\
\mathcal{K}_{2}\left(\Phi_{\left(n_{1}, n_{2}\right)}\right)=: & \Phi_{\left(n_{1}, n_{2}+1\right)}+\sum P_{i,\left(n_{1}, n_{2}+1\right)}^{\Phi} \frac{\partial}{\partial x_{i}} W_{k}
\end{array}
$$

where $\Phi_{\left(n_{1}, n_{2}\right)} \in \mathcal{R}_{x}^{(k)}$. Note that these expansions are in general not unique, and this, in fact, corresponds to choosing a gauge (that is, by writing $\mathcal{K}_{1}\left(\Phi_{i} \cdot \Phi_{j}\right) \equiv \Gamma_{i j}^{k} \Phi_{k}$, we see that it amounts to a gauge choice for the Gauß-Manin connection $\Gamma$ [34]). Therefore the precise image of a given $\Phi$ under the map $\mathcal{K}_{1}$ or $\mathcal{K}_{2}$ depends, in general, on this gauge. By choosing the $P_{i}^{\Phi}$ appropriately, the $\mathcal{K}_{i}$ can always be made to act between the $W_{3}$ eigen-polynomials $\Phi^{l, m}$.

By definition, the $\mathcal{K}_{i}$ map physical operators into physical ones and unphysical (null) operators into unphysical ones, and this allows to recursively determine whether any given polynomial $\Phi^{l, m}$ describes a physical state or not. For $l \geq t$, it is easy to see that one can always choose the $P_{i}^{\Phi}$ such that

$$
\mathcal{K}_{1,2}: \Phi^{l, m}\left(x_{1}, x_{2}\right) \longrightarrow \Phi^{l-t, m}\left(x_{1}, x_{2}\right)
$$


so that all operators with $l \geq t$ can be related to operators in the strip $l<t$. The action of the $\mathcal{K}_{i}$ on the operators with $l<t$ is, on the other hand, in general quite complicated, but can be deduced case by case. The result from such an analysis is that all $\Phi^{l, m}$ become physical operators after coupling to $W$-gravity, except for

$$
\begin{aligned}
& \left\{\Phi^{(l+1) t-m-2, m}\left(x_{1}, x_{2}\right), \quad l=0,1,2, \ldots, m=0,1,2, \ldots(l+1) t-2\right\} \\
& \left\{\Phi^{(l+1) t-1, m}\left(x_{1}, x_{2}\right), \Phi^{l,(m+1) t-1}\left(x_{1}, x_{2}\right), \quad l, m=0,1,2, \ldots\right\},
\end{aligned}
$$

which remain null. This list corresponds to eq. (1.3) for one variable. The spectrum for $t=7$ is depicted in Fig.3, which is the two-variable analog of Fig.1.

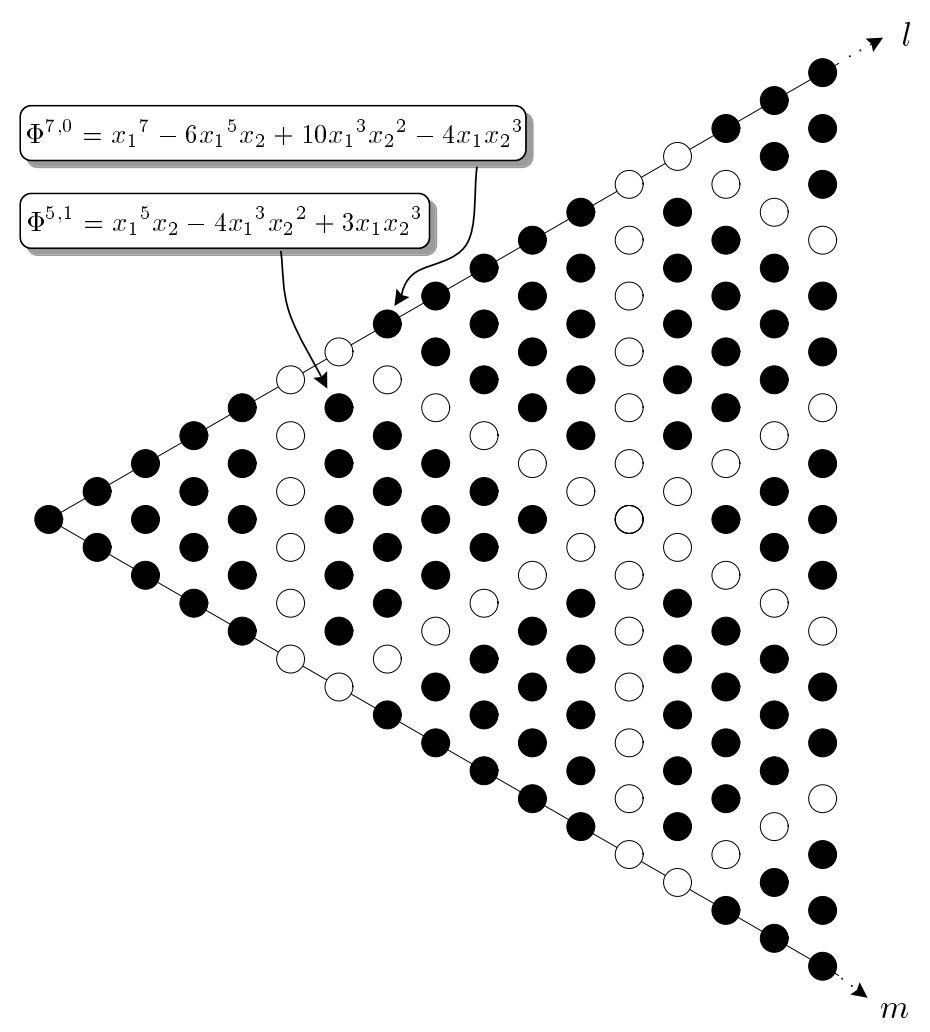

Fig. 3 Gravitational chiral ring associated with the topological $W_{3}$ minimal model $\mathrm{CP}_{2,4}$, represented in terms of Landau-Ginzburg polynomials $\Phi^{l, m}\left(x_{1}, x_{2}\right)$. The projection on the $m$-axis gives the $U(1)$ charge $(2.16)$. The open dots denote null fields. The leftmost triangle of black dots represents the primary chiral ring $\mathcal{R}_{x}^{(4)}$ of the matter model, while the other black dots represent their gravitational descendants. The descendant generators are linear combinations of the two operators indicated. 
The interpretation of the physical states with $l+m>k \equiv t-3$ is, of course, that they are the gravitational descendants of the matter chiral ring $\mathcal{R}_{x}^{(k)}$, and it is easy to see that the $\mathcal{K}_{i}(\Phi)$ are linear combinations of inverses of gravitational descendant generators $\sigma_{1}, \sigma_{2}$. More specifically, consider

$$
\begin{aligned}
\sigma_{1} \equiv \sigma_{1}(1):=\Phi^{t, 0} & \equiv \frac{\partial}{\partial x_{1}} W_{k+1}\left(x_{1}, x_{2}\right) \\
& =\left(x_{1} \frac{\partial}{\partial x_{1}}+x_{2} \frac{\partial}{\partial x_{2}}\right) W_{k}\left(x_{1}, x_{2}\right),
\end{aligned}
$$

where in the second line we used (2.13). The expression in the second line is the same as the expression for the dilaton in terms of LG fields that was found by Lossev [14]. Indeed, $\mathcal{K}_{1}$ is identical to the recursion operator of [14] that satisfies

$$
\mathcal{K}_{1}\left(\sigma_{1}(\Phi)\right)=2 \Phi
$$

which is solved by

$$
\sigma_{1}(\Phi) \cong \frac{\partial}{\partial x_{1}} W_{k}\left(x_{1}, x_{2}\right)\left(\int^{x_{1}} d x_{1}^{\prime} \Phi\right)+\frac{\partial}{\partial x_{2}} W_{k}\left(x_{1}, x_{2}\right)\left(\int^{x_{2}} d x_{2}^{\prime} \Phi\right) .
$$

$\mathcal{K}_{1}$ also appears in the puncture operator contact term. Specifically, noting that for $l, m \geq 0$ :

$$
\mathcal{K}_{1}\left(\Phi^{t+l, m}\right)=(l+m+2) \Phi^{l, m},
$$

one gets

$$
\int_{|z|<\epsilon} \Phi(z)\left|\Phi^{t+l, m}\right\rangle=\Phi(0)\left|\mathcal{K}_{1}\left(\Phi^{t+l, m}\right)\right\rangle=(l+m+2)\left|\left(\Phi \cdot \Phi^{l, m}\right)\right\rangle,
$$

and this leads, essentially, to a Virasoro algebra [32]. It would be very interesting to find a $W$-geometric interpretation of the other operator, $\mathcal{K}_{2}$, in relation with contact terms, but this is beyond our present scope.

As for the other gravitational descendant generator, $\sigma_{2}$, we find that using identities like (2.13) one has for $l+m \leq k$ :

$$
\mathcal{K}_{1}\left(\Phi^{t-l-2, m+l+1}\right)=-(m+1) \Phi^{l, m} .
$$

(In terms of Fig.2, the operators $\Phi^{t+l, m}$ and $\Phi^{t-l-2, m+l+1}$ live in the two triangles that are pointed to by the arrows.) Therefore the linear combinations

$$
\sigma_{2}\left(\Phi^{l, m}\right): \equiv(l+m+2) \Phi^{t-l-2, m+l+1}+(m+1) \Phi^{t+l, m}
$$


obey

$$
\mathcal{K}_{1}\left(\sigma_{2}\left(\Phi^{l, m}\right)\right)=0
$$

On the other hand, one can check that

$$
\mathcal{K}_{2}\left(\sigma_{2}\left(\Phi^{l, m}\right)\right)=v_{2} \Phi^{l, m}
$$

with some generically non-zero coefficient $v_{2}$. This means that although $\sigma_{2}\left(\Phi^{l, m}\right)$ are null operators in ordinary topological gravity, they become physical if one imposes the additional $W_{3}$ equivariance condition, $b_{2} \Phi \cong 0$. Hence, these operators can be viewed as LG representatives of the extra gravitational descendants of $\mathcal{R}_{x}^{(k)}$ that are due to the $W_{3}$ extension of topological gravity. In particular, the $W_{3}$ descendant generator is

$$
\sigma_{2} \equiv \sigma_{2}(1)=\Phi^{t, 0}+2 \Phi^{t-2,1}=\left(x_{1} \frac{\partial}{\partial x_{1}}+3 x_{2} \frac{\partial}{\partial x_{2}}\right) W_{k}\left(x_{1}, x_{2}\right) .
$$

One expects that $\sigma_{1,2}$ are LG representatives of the basic operators of topological $W_{3}$-gravity, and we like to find the precise relationship by making use of an argument similar to the one of ref. [11]. For this, note that in analogy to ordinary gravity [10,20], the non-trivial cohomology in pure topological $W$-gravity is (supposedly completely) generated by

$$
\begin{aligned}
\gamma_{i}^{0} & =\frac{1}{2}\left\{Q_{S}-\bar{Q}_{S},\left\{Q_{V}, \phi_{i}\right\}\right\} \\
& =: \frac{1}{2}\left\{Q_{S}, \Lambda^{\gamma_{i}}\right\}-\text { c.c }, \quad i=1,2 .
\end{aligned}
$$

(These operators are non-trivial because the Liouville fields $\phi_{i}$ do not create states in the physical Hilbert space.) From the BRST operator (3.13) we have

$$
\begin{aligned}
\Lambda^{\gamma_{1}} & =-\frac{1}{2} \partial c_{1}-\frac{19}{46} \partial^{2} c_{2}+\left(c_{1} \partial \phi_{1}+\ldots\right) \\
\Lambda^{\gamma_{2}} & =-\partial c_{1}+\frac{4}{23} \partial^{2} c_{2}+\left(c_{1} \partial \phi_{2}+\ldots\right)
\end{aligned}
$$

where the parentheses indicate terms that are not important here. For convenience, let us first introduce

$$
\widetilde{\sigma}_{1}=(18-5 t) \sigma_{1}+\left(t^{2}-t-6\right) \sigma_{2}
$$

and note that

$$
\begin{aligned}
& \mathcal{K}_{1}\left(\widetilde{\sigma}_{1}\right)=2(18-5 t) \\
& \mathcal{K}_{2}\left(\widetilde{\sigma}_{1}\right)=0 \\
& \mathcal{K}_{1}\left(\sigma_{2}\right)=0 \\
& \mathcal{K}_{2}\left(\sigma_{2}\right)=2 \frac{5 t-18}{(t+2)(t-4)} \alpha_{m}
\end{aligned}
$$


as well as

$$
\begin{aligned}
& \left(b_{1}\right)_{0} \cdot \Lambda^{\gamma_{1}}=-\frac{1}{2} \\
& \left(b_{2}\right)_{0} \cdot \Lambda^{\gamma_{1}}=-\frac{19}{23} \\
& \left(b_{1}\right)_{0} \cdot \Lambda^{\gamma_{2}}=-1 \\
& \left(b_{2}\right)_{0} \cdot \Lambda^{\gamma_{2}}=\frac{8}{23} .
\end{aligned}
$$

Then, observing that the Liouville and ghost parts in $G_{t o t}^{-}, U_{t o t}^{-}$do not contribute to the constant terms that we are looking at and that $S \gamma_{i}^{0} S^{-1}=\gamma_{i}^{0}$, we see the following equivariance conditions to be satisfied:

$$
\begin{aligned}
& \left\{\left(b_{1}+G_{t o t}^{-}\right)_{0}, \alpha_{1}\left(8 \Lambda^{\gamma_{1}}+19 \Lambda^{\gamma_{2}}\right)-{\widetilde{\sigma^{1}}}^{\tilde{\sigma}_{1}}=0\right. \\
& \left\{\left(b_{2}+U_{t o t}^{-}\right)_{0}, \alpha_{1}\left(8 \Lambda^{\gamma_{1}}+19 \Lambda^{\gamma_{2}}\right)-{\widetilde{\sigma^{\prime}}}\right\} \equiv 0
\end{aligned}
$$

where $\alpha_{1}=\frac{2}{23}(5 t-18)$, and

$$
\begin{aligned}
& \left\{\left(b_{1}+G_{t o t}^{-}\right)_{0}, \alpha_{2}\left(2 \Lambda^{\gamma_{1}}-\Lambda^{\gamma_{2}}\right)-\Lambda^{\sigma_{2}}\right\} \equiv 0 \\
& \left\{\left(b_{2}+U_{t o t}^{-}\right)_{0}, \alpha_{2}\left(2 \Lambda^{\gamma_{1}}-\Lambda^{\gamma_{2}}\right)-\Lambda^{\sigma_{2}}\right\}=0
\end{aligned}
$$

where $\alpha_{2}=\frac{18-5 t}{(t+2)(t-4)} \alpha_{m}$. This means that we have, in equivariant cohomology, the following relationship between the two representations of the gravitational ring generators:

$$
\begin{aligned}
& \widetilde{\sigma}_{1}\left(x_{1}, x_{2}\right) \cong \alpha_{1}\left(8 \gamma_{1}^{0}+19 \gamma_{2}^{0}\right) \\
& \sigma_{2}\left(x_{1}, x_{2}\right) \cong \alpha_{2}\left(2 \gamma_{1}^{0}-\gamma_{2}^{0}\right) .
\end{aligned}
$$

Because of the polynomial ring structure, it is quite clear that similar equivalences should hold also for powers of the $\sigma_{i}$.

\section{Conclusions}

We investigated how topological $W$-gravity can be realized in terms of twisted $N=2$ Landau-Ginzburg theory. We showed that by using only information that is intrinsic to a given LG "matter" model, one can recover the chiral ground ring of topological $W$-gravity, and generate pictures like Fig.4. We considered as example the special case of $W_{3}$, but it is fairly obvious that our methods should work for general $W_{n}$ in an analogous way (and probably for even more general LG theories as well). 


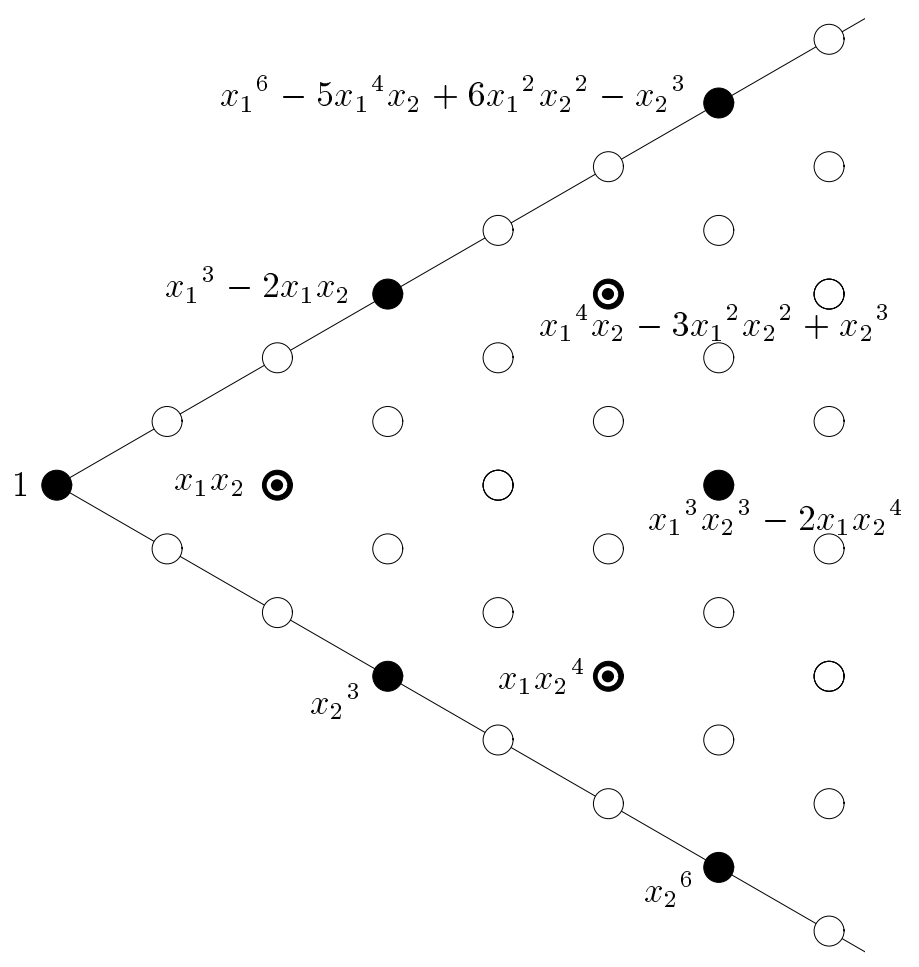

Fig. 4 Polynomial representation of pure $W_{3}$-gravity. The spectrum corresponds to the "trivial" superpotential $W_{0}=\frac{1}{3} x_{1}{ }^{3}-x_{1} x_{2}$ with vanishing $N=2$ central charge. The marked dots denote physical states that were unexpected from previous work.

More specifically, we first showed how one can construct the extended (twisted) $N=2$ current algebra in terms of Landau-Ginzburg data, which are the LG fields and their superpotential. We then used these currents to describe the effect of requiring equivariant cohomology on the LG spectrum. The precise form of the gravity sector, and hence of the BRST operator, is not important here, since it decouples. The only important ingredient is eq. (4.3), which ensures that the cohomology can be represented entirely in the LG sector. The precise form of the chiral algebra currents then directly translates into which LG polynomials become physical and which stay null after coupling to topological $W$-gravity. 
We find this simple algorithm quite remarkable, since the polynomial LG spectrum obtained in this way carries implicitly a lot of mathematical information, for example about the structure of singular vectors of type $(1, t) W_{3}$ minimal models, and about representation theory of affine algebras.

Our results also indicate that the spectrum of $W$-gravity coupled to matter contains more states than previously thought. In refs. [2][35], the $W_{3}$ chiral ground ring for $t=k+3$ was found to contain the following operators:

$$
\mathcal{R}_{x, \gamma}^{(k)}=\mathcal{R}_{x}^{(k)}\left(x_{1}, x_{2}\right) \otimes\left\{\left(\sigma_{1}\right)^{r_{1}}\left(\sigma_{2}\right)^{r_{2}}, \quad r_{1}, r_{2}=1,2, \ldots\right\},
$$

where $\sigma_{i}$ denote gravitational generators with $U(1)$ charges

$$
J_{0}\left(\sigma_{i}\right)=i .
$$

In contrast, we find in the present paper a ring generator $\sigma_{2}$ already at level one and not at level two,

$$
J_{0}\left(\sigma_{i}\right)=1 .
$$

The new spectrum contains the old one, and to be explicit, we marked in Fig.4 the new additional states.

Note that our present findings do not invalidate the results of refs. [2,35], where one was looking for ring generators with the simplest possible structure (that turned out be be already quite complicated!). It would be very interesting to understand the emergence of the additional states in terms of the cohomology of the $W_{3}$-mattergravity system.

\section{Acknowledgements}

We thank Erik Verlinde for discussions, and Kris Thielemans for his Mathematica package SOPEN2defs.m. 


\section{Appendix A. Superspace conventions and the $N=2 \quad W_{3}$ alge- bra}

We use $N=2$ superspace with coordinates $\left\{z, \theta^{+}, \theta^{-}\right\}$. The derivatives are defined as

$$
\begin{aligned}
D_{ \pm} & \equiv D^{\mp} \equiv \frac{\partial}{\partial \theta^{ \pm}}-\frac{1}{2} \theta^{\mp} \frac{\partial}{\partial z} \\
\partial & \equiv \frac{\partial}{\partial z} \\
D_{+-} & \equiv-\frac{1}{2}\left[D_{+}, D_{-}\right],
\end{aligned}
$$

such that

$$
\left\{D_{+}, D_{-}\right\}=-\partial
$$

From

$$
\frac{1}{2 \pi i} \oint d Z_{1} \frac{\theta_{12}^{+} \theta_{12}^{-}}{z_{12}} \Phi\left(Z_{1}\right)=\Phi\left(Z_{2}\right)
$$

one deduces the $N=2$ Taylor expansion

$$
\Phi\left(Z_{1}\right)=\sum_{n \geq 0} \frac{z_{12}^{n}}{n !} \partial^{n}\left(1+\theta_{12}^{+} D_{+}+\theta_{12}^{-} D_{-}+\theta_{12}^{+} \theta_{12}^{-} D_{+-}\right) \Phi\left(Z_{2}\right),
$$

where

$$
\begin{aligned}
& \theta_{12}^{ \pm} \equiv \theta_{1}^{ \pm}-\theta_{2}^{ \pm} \\
& z_{12} \equiv z_{1}-z_{2}+\frac{1}{2} \theta_{1}^{+} \theta_{2}^{-}+\frac{1}{2} \theta_{1}^{-} \theta_{2}^{+} .
\end{aligned}
$$

The super energy momentum tensor $\mathcal{T}$ is an unconstrained superfield which satisfies the OPE:

$$
\mathcal{T}\left(Z_{1}\right) \mathcal{T}\left(Z_{2}\right)=\frac{c}{3} \frac{1}{z_{12}^{2}}+\left(\frac{\theta_{12}^{+} \theta_{12}^{-}}{z_{12}^{2}}+\frac{\theta_{12}^{-}}{z_{12}} D_{-}-\frac{\theta_{12}^{+}}{z_{12}} D_{+}+\frac{\theta_{12}^{+} \theta_{12}^{-}}{z_{12}} \partial\right) \mathcal{T}\left(Z_{2}\right) .
$$

An unconstrained superfield $\mathcal{O}$ is primary with conformal dimension $h$ and $U(1)$ charge $q$ if

$$
\mathcal{T}\left(Z_{1}\right) \mathcal{O}\left(Z_{2}\right)=\left(h \theta_{12}^{+} \theta_{12}^{-} \frac{1}{z_{12}^{2}}+\frac{\theta_{12}^{-}}{z_{12}} D_{-}-\frac{\theta_{12}^{+}}{z_{12}} D_{+}+\frac{\theta_{12}^{+} \theta_{12}^{-}}{z_{12}} \partial+q \frac{1}{z_{12}}\right) \mathcal{O}\left(Z_{2}\right) .
$$

The superfield $\mathcal{O}$ is said to be chiral if it satisfies $D_{+} \mathcal{O}=0$ and consistency of eq. (A.3)with this requires that $h=q / 2$. Similarly, one can define an anti-chiral superfield $\mathcal{O}$ which satisfies $D_{-} \mathcal{O}=0$ and which has $h=-q / 2$. 
In this paper, we mainly deal with the $N=2 W_{3}$ algebra [24]. This algebra is generated by the super energy momentum tensor and one additional unconstrained superfield $\mathcal{W}$. It is primary with conformal dimension 2 and vanishing $U(1)$ charge. The operator product expansion of $\mathcal{W}$ with itself is rather involved, but we give it here to correct some slight misprints of the component results given in the literature:

$$
\begin{aligned}
& \mathcal{W}\left(Z_{1}\right) \mathcal{W}\left(Z_{2}\right)=\frac{1}{z_{12}^{4}}\left\{\frac{c}{6}+\theta_{12}^{+} \theta_{12}^{-} \mathcal{T}\right\}-\frac{1}{z_{12}^{3}}\left\{\theta_{12}^{+} D_{+} \mathcal{T}-\theta_{12}^{-} D_{-} \mathcal{T}-\theta_{12}^{+} \theta_{12}^{-} \partial \mathcal{T}\right\}+ \\
& \frac{1}{z_{12}^{2}}\left\{( 1 - c ) ^ { - 1 } \left(-\frac{2 c}{3} D_{+-} \mathcal{T}+\mathcal{T} \mathcal{T}+\theta_{12}^{-}\left(\frac{3-2 c}{3} \partial D_{-} \mathcal{T}+\mathcal{T} D_{-} \mathcal{T}\right)-\right.\right. \\
& \theta_{12}^{+}\left(\frac{3-2 c}{3} \partial D_{+} \mathcal{T}-\mathcal{T} D_{+} \mathcal{T}\right)+(4(3-2 c)(6+c))^{-1} \theta_{12}^{+} \theta_{12}^{-}\left(3(10 c-24) c \partial D_{+-} \mathcal{T}+\right. \\
& 4\left(36-9 c+8 c^{2}\right) \mathcal{T} D_{+-} \mathcal{T}-12(3+4 c) \mathcal{T} \mathcal{T} \mathcal{T}+12(12-5 c) c D_{-} \mathcal{T} D_{+} \mathcal{T}+ \\
& \left.\left.2\left(18-15 c+2 c^{2}+2 c^{3}\right) \partial^{2} \mathcal{T}\right)\right)+\alpha\left(\mathcal{W}+\frac{1}{2} \theta_{12}^{-} D_{-} \mathcal{W}+\frac{1}{2} \theta_{12}^{+} D_{+} \mathcal{W}-\right. \\
& \left.\left.\theta_{12}^{+} \theta_{12}^{-} 3(4(12-5 c))^{-1}\left(28 \mathcal{T} \mathcal{W}-2(8-c) D_{+-} \mathcal{W}\right)\right)\right\}+ \\
& \frac{1}{z_{12}}\left\{(1-c)^{-1} \partial \mathcal{T} \mathcal{T}-c(3(1-c))^{-1} \partial D_{+-} \mathcal{T}+\right. \\
& \theta_{12}^{+}(2(1-c)(3-2 c)(6+c))^{-1}\left(-c\left(9-3 c+c^{2}\right) \partial^{2} D_{+} \mathcal{T}+6(3+4 c) \mathcal{T} \mathcal{T} D_{+} \mathcal{T}+\right. \\
& \left.18(1-c) c D_{+-} \mathcal{T} D_{+} \mathcal{T}+2\left(18-24 c-c^{2}\right) \partial D_{+} \mathcal{T} \mathcal{T}-3(1-c)(6-c) \partial \mathcal{T} D_{+} \mathcal{T}\right)+ \\
& \theta_{12}^{-}(2(1-c)(3-2 c)(6+c))^{-1}\left(\frac{1}{2} c\left(9+3 c+2 c^{2}\right) \partial^{2} D_{-} \mathcal{T}-6(3+4 c) \mathcal{T} \mathcal{T} D_{-} \mathcal{T}-\right. \\
& \left.18(1-c) c D_{-} \mathcal{T} D_{+-} \mathcal{T}+2\left(18-24 c-c^{2}\right) \partial D_{-} \mathcal{T} \mathcal{T}-3(1-c)(6-c) \partial \mathcal{T} D_{-} \mathcal{T}\right)+ \\
& \theta_{12}^{+} \theta_{12}^{-}(2(1-c)(3-2 c)(6+c))^{-1}\left(4(12-5 c) c \partial D_{-} \mathcal{T} D_{+} \mathcal{T}+\right. \\
& 8\left(9-3 c+c^{2}\right) \partial D_{+-} \mathcal{T} \mathcal{T}-4(12-5 c) c \partial D_{+} \mathcal{T} D_{-} \mathcal{T}+4 c(3+4 c) \partial \mathcal{T} D_{+-} \mathcal{T}- \\
& \left.12(3+4 c) \partial \mathcal{T} \mathcal{T} \mathcal{T}-\frac{1}{3}\left(18+3 c+2 c^{2}-2 c^{3}\right) \partial^{3} \mathcal{T}\right)+\alpha\left(\frac{1}{2} \partial \mathcal{W}+\right. \\
& \theta_{12}^{+}(2(12-5 c)(3+c))^{-1}\left(3(c-1)(6-c) \partial D_{+} \mathcal{W}+6(15-c) \mathcal{T} D_{+} \mathcal{W}-\right. \\
& \left.54(1-c) D_{+} \mathcal{T} \mathcal{W}\right)+\theta_{12}^{-}(2(12-5 c)(3+c))^{-1}\left(3(c-1)(6-c) \partial D_{-} \mathcal{W}-\right. \\
& \left.6(15-c) \mathcal{T} D_{-} \mathcal{W}+54(1-c) D_{-} \mathcal{T} \mathcal{W}\right)+\theta_{12}^{+} \theta_{12}^{-}(2(12-5 c)(3+c))^{-1} \times \\
& \left(2(15-c) c \partial D_{+-} \mathcal{W}-18(6+c) \mathcal{T} \partial \mathcal{W}+6(12-5 c) D_{-} \mathcal{T} D_{+} \mathcal{W}+\right. \\
& \left.\left.\left.6(12-5 c) D_{+} \mathcal{T} D_{-} \mathcal{W}-12(3+4 c) \partial \mathcal{T} \mathcal{W}\right)\right)\right\}
\end{aligned}
$$

where

$$
\alpha=\frac{\sqrt{2}(3+c)(12-5 c)}{\sqrt{3(3-2 c)(-15+c)(-1+c)(6+c)}} .
$$




\section{References}

[1] B. Gato-Rivera and A.M. Semikhatov, Phys. Lett. B293 (1992) 72.

[2] M. Bershadsky, W. Lerche, D. Nemeschansky and N.P. Warner, Nucl. Phys. $\underline{B 401}$ (1993) 304.

[3] For a review, see eg., W. Lerche, Chiral Rings and Integrable Systems for Models of Topological Gravity, to appear in the Proceedings of Strings '93, Berkeley, preprint CERN-TH.7128/93, hep-th/9401121.

[4] C. Vafa, Mod. Phys. Let. $\underline{A 6}$ (1991) 337.

[5] E. Witten, Comm. Math. Phys. 117 (1988) 353; Comm. Math. Phys. 118 (1988) 411; Nucl. Phys. B340 (1990) 281.

[6] T. Eguchi and S. Yang, Mod. Phys. Let. $\underline{A 4}$ (1990) 1693.

[7] M. Douglas, Phys. Lett. 238B (1990) 176.

[8] R. Dijkgraaf, E. Verlinde and H. Verlinde, Nucl. Phys. B352 (1991) 59.

[9] K. Li, Nucl. Phys. B354 (1991) 711; Nucl. Phys. B354 (1991)725.

[10] E. Witten, Nucl. Phys. B340 (1990) 281; R. Dijkgraaf and E. Witten, Nucl. Phys. $\underline{B 342}$ (1990) 486; J. Distler, Nucl. Phys. $\underline{B 342}$ (1990) 523; E. and H. Verlinde, Nucl. Phys. B348 (1991) 457; R. Dijkgraaf and E. and H. Verlinde, Nucl. Phys. $\underline{B 348}$ (1991) 435; For a review, see: R. Dijkgraaf, Intersection theory, integrable hierarchies and topological field theory, preprint IASSNS-HEP-91/91.

[11] T. Eguchi, H. Kanno, Y. Yamada and S.-K. Yang, Phys. Lett. B305 (1993) 235.

[12] J. Distler and P. Nelson, Phys. Rev. Lett. 66 (1991) 1955.

[13] C. Vafa and N.P. Warner, Phys. Lett. $\underline{218 B}$ (1989) 51; E. Martinec, Phys. Lett. $\underline{217 B}(1989) 431$.

[14] A. Lossev, Descendants constructed from matter field and K. Saito higher residue pairing in Landau-Ginzburg theories coupled to topological gravity, preprint TPIMINN-92-40-T.

[15] W. Lerche, Generalized Drinfeld-Sokolov Hierarchies, Quantum Rings, and WGravity, preprint CERN-TH.6988/93. 
[16] M. Bershadsky, W. Lerche, D. Nemeschansky and N.P. Warner, Phys. Lett. B292 (1992) 35; E. Bergshoeff, A. Sevrin and X. Shen, Phys. Lett. 296 (1992) 95; J. de Boer and J. Goeree, Nucl. Phys. $\underline{B 405}$ (1993) 669.

[17] Y. Kazama and H. Suzuki, Nucl. Phys. B321 (1989) 232.

[18] W. Lerche, C. Vafa and N.P. Warner, Nucl. Phys. B324 (1989) 427.

[19] D. Gepner, A comment on the chiral algebras of quotient superconformal field theories, preprint PUPT-1130; S. Hosono and A. Tsuchiya, Comm. Math. Phys. $\underline{136}$ (1991) 451.

[20] K. Li, Phys. Lett. $\underline{B 251}$ (1990) 54, Nucl. Phys. $\underline{B 346}$ (1990) 329, Linear $W_{N^{-}}$ gravity, preprint CALT-68-1724; H. Lu, C.N. Pope and X. Shen, Nucl. Phys. $\underline{B 366}$ (1991) 95; S. Hosono, Algebraic definition of topological W-gravity, preprint UT588; H. Kunitomo, Prog. Theor. Phys. 86 (1991) 745.

[21] P. Fré, L. Girardello, A. Lerda and P. Soriani, Nucl. Phys. B387 (1992) 333.

[22] E. Witten, On the Landau-Ginzburg description of $N=2$ minimal models, preprint IASSNS-HEP-93-1.

[23] D. Nemeschanksy and N. Warner, Refining the elliptic genus, preprint USC94/002.

[24] K. Ito, Phys. Lett. $\underline{B 259}$ (1991) 73; Nucl. Phys. $\underline{B 370}$ (1992) 123; D. Nemeschansky and S. Yankielowicz, N=2 W-algebras, Kazama-Suzuki models and DrinfeldSokolov reduction, preprint USC-91-005A; L. Romans, Nucl. Phys. B369 (1992) 403.

[25] P. Fendley, W. Lerche, S. Mathur and N.P. Warner, Nucl. Phys. B348 (1991) 66; W. Lerche and N.P. Warner, Nucl. Phys. B358 (1991) 571.

[26] D. Gepner, Comm. Math. Phys. 141 (1991) 381.

[27] A. Boresch, K. Landsteiner, W. Lerche and A. Sevrin, Topological strings from Hamiltonian reduction, to appear.

[28] J. de Boer and T. Tjin, The relation between quantum $W$ algebras and Lie algebras, preprint THU-93-05; A. Sevrin and W. Troost, Phys. Lett. $\underline{315}$ (1993) 304.

[29] L. Romans, Nucl. Phys. B352 (1991) 829.

[30] V. Kac, Comm. Math. Phys. $\underline{53}$ (1977) 31; L. Frappat, E. Ragouchy and P. Sorba, Comm. Math. Phys. 157 (1993) 499. 
[31] P. Goddard and A. Schwimmer, Phys. Lett. 214 (1988) 447.

[32] E. and H. Verlinde, in [10].

[33] J. Distler and P. Nelson, Comm. Math. Phys. 138 (1991) 273.

[34] K. Saito, J. Fac. Sci. Univ. Tokyo Sec. IA.28 (1982) 775; M. Noumi, Tokyo. J. Math. 7 (1984) 1; B. Blok and A. Varchenko, Topological conformal field theories and the flat coordinates, preprint IASSNS-HEP-91/5; S. Cecotti and C. Vafa, Nucl. Phys. B367 (1991) 359; W. Lerche, D. Smit and N. Warner, Nucl. Phys. $\underline{B 372}$ (1992) 87.

[35] P. Bouwknegt, J. McCarthy and K. Pilch, Lett. Math. Phys. 29 (1993) 91; On the BRST structure of $W_{3}$-gravity coupled to $c=2$ matter, preprint USC-93/14; On the $W$-gravity spectrum and its G-structure, preprint USC-93/27. 